\title{
SHAPE OPTIMIZATION OF CONTACT PROBLEMS WITH SLIP RATE DEPENDENT FRICTION
}

\author{
A. Myśliński ${ }^{1}$ \\ ${ }^{1}$ System Research Institute, Warsaw, Poland, myslinsk@ibspan.waw.pl
}

\begin{abstract}
This paper deals with the formulation of a necessary optimality condition for a shape optimization problem of a viscoelastic body in unilateral dynamic contact with a rigid foundation. The contact with Coulomb friction is assumed to occur at a portion of the boundary of the body. The contact condition is described in velocities. The friction coefficient is assumed to be bounded and Lipschitz continuous with respect to a slip velocity. The evolution of the displacement of the viscoelastic body in unilateral contact is governed by a hemivariational inequality of the second order. The shape optimization problem for a viscoelastic body in contact consists in finding, in a contact region, such shape of the boundary of the domain occupied by the body that the normal contact stress is minimized. It is assumed, that the volume of the body is constant. Using material derivative method, we calculate the directional derivative of the cost functional and we formulate a necessary optimality condition for this problem.
\end{abstract}

keywords: dynamic unilateral problem, shape optimization, necessary optimality condition

\section{Introduction}

This paper deals with the formulation of a necessary optimality condition for a shape optimization problem of a viscoelastic body in unilateral dynamic contact with a rigid foundation. The contact with a given Coulomb friction $[2,3]$ is assumed to occur at a portion of the boundary of the body. The contact condition is described in velocities. Usually $[2,3]$ this contact problem is considered either with a constant or suitable small functional friction coefficient depending on spatial variables. Numerous experiments indicate $[5,7]$, that in the study of many frictional processes (stick - sleep motions, earthquake modelling, etc.) the friction coefficient has to be considered variable during the slip. The evolution of the body in the unilateral contact is described by a variational inequality of the second order $[2,4]$. Under the assumption that the friction coefficient is bounded and Lipschitz continuous with respect to the sliding velocity, the

Please use the following format when citing this chapter:

Myśliński, A., 2006, in IFIP International Federation for Information Processing, Volume 202, Systems, Control, Modeling and Optimization, eds. Ceragioli, F., Dontchev, A., Furuta, H., Marti, K., Pandolfi, L., (Boston: Springer), pp. 285-295. 
existence of solutions to the viscoelastic dynamic contact problems is shown in $[3,5]$. For regularity result with respect to time variable see [7].

The shape optimization problem for a viscoelastic body in unilateral contact consists in finding, in a contact region, such shape of the boundary of the domain occupied by the body that the normal contact stress is minimized. It is assumed that the volume of the body is constant. Shape optimization of static contact problems was considered, among others, in $[4,11]$. The necessary optimality conditions for shape optimization of dynamic contact problems were formulated in $[6,9]$.

In this paper we study this shape optimization problem for a viscoelastic body in unilateral dynamical contact with friction coefficient bounded and Lipschitz dependent on a slip rate. Assuming small friction coefficient and suitable regularity of data it can be shown [3] that the solution to dynamic contact problem is enough regular to differentiate it with respect to a parameter. Using material derivative method [11], we calculate the directional derivative of the cost functional and we formulate a necessary optimality condition for this problem. The present paper extends authors results contained in [9].

The following notation will be employed: $\Omega \subset R^{2}$ will denote the bounded domain with Lipschitz continuous boundary $\Gamma$. The time variable will be denoted by $t$ and the time interval $I=(0, \mathcal{T}), \mathcal{T}>0$. By $H^{k}(\Omega), k \in(0, \infty)$, we will denote the Sobolev space of functions having derivatives in all directions of the order $k$ belonging to $L^{2}(\Omega)$ [1]. For an interval $I$ and a Banach space $B, L^{p}(I ; B), p \in[1, \infty)$, denotes the usual Bochner space [3]. $u_{t}=d u / d t$ and $u_{t i t}=d^{2} u / d t^{2}$ will denote first and second order derivatives, respectively, with respect to $t$ of function $u . u_{t N}$ and $u_{t T}$ will denote normal and tangential components, respectively, of function $u_{t} . Q=I \times \Omega, \gamma_{i}=I \times \Gamma_{i}, i=1,2,3$, where $\Gamma_{i}$ are pieces of the boundary $\Gamma$.

\section{Contact problem formulation}

Consider deformations of a viscoelastic body occupying domain $\Omega \subset R^{2}$. The boundary $\Gamma$ of domain $\Omega$ is Lipschitz continuous. The body is subject to body forces $f=\left(f_{1}, f_{2}\right)$. Moreover surface tractions $p=\left(p_{1}, p_{2}\right)$ are applied to a portion $\Gamma_{1}$ of the boundary $\Gamma$. We assume that the body is clamped along the portion $\Gamma_{0}$ of the boundary $\Gamma$ and that the contact conditions are prescribed on the portion $\Gamma_{2}$ of the boundary $\Gamma$. Moreover $\Gamma_{i} \cap \Gamma_{j}=\emptyset, i \neq j, i, j=0,1,2$, $\Gamma=\bar{\Gamma}_{0} \cup \bar{\Gamma}_{1} \cup \bar{\Gamma}_{2}$.

Let us denote by $u=\left(u_{1}, u_{2}\right), u=u(t, x), x \in \Omega, t \in[0, \mathcal{T}], \quad \mathcal{T}>0$ the displacement of the body and by $\sigma=\left\{\sigma_{i j}(u(t, x))\right\}, i, j=1,2$, the stress field in the body. We shall consider viscoelastic bodies obeying Kevin - Voigt law $[2-4]$ :

$$
\sigma_{i j}(u)=c_{i j k l}^{0}(x) e_{k l}(u)+c_{i j k l}^{1}(x) e_{k l}\left(u_{t}\right) x \in \Omega,
$$


where $e_{k l}(u)=\frac{1}{2}\left(u_{k, l}+u_{l, k}\right), i, j, k, l=1,2, u_{k, l}=\partial u_{k} / \partial x_{l}$. The summation convention over repeated indices [2] is used throughout the paper. $c_{i j k l}^{0}(x)$ and $c_{i j k l}^{1}(x), i, j, k, l=1,2$, are components of elasticity and viscoelasticity tensors. It is assumed that elements $c_{i j k l}^{0}$ and $c_{i j k l}^{1}$ satisfy usual symmetry, boundedness and ellipticity conditions [2-4]. The displacement field $u$ is a solution of the linear viscoelastic system [2]:

$$
u_{t t i}-\sigma_{i j}(u(x))_{, j}=f_{i}(x), \quad(t, x) \in(0, \mathcal{T}) \times \Omega, \quad i, j=1,2,
$$

where $\sigma_{i j}(x)_{, j}=\partial \sigma_{i j}(x) / \partial x_{j}, i, j=1,2$. There are given the following boundary conditions for $i, j=1,2$ :

$$
u_{i}(x)=0 \text { on }(0, \mathcal{T}) \times \Gamma_{0}, \text { and } \sigma_{i j}(x) n_{j}=p_{i} \text { on }(0, \mathcal{T}) \times \Gamma_{1},
$$

as well as the contact and friction conditions on the boundary $(0, \mathcal{T}) \times \Gamma_{2}$,

$$
\begin{array}{r}
u_{t N} \leq 0, \quad \sigma_{N} \leq 0, \quad u_{t N} \sigma_{N}=0 \\
u_{t T}=0 \Rightarrow\left|\sigma_{T}\right| \leq \mathcal{F}\left|\sigma_{N}\right|, \\
u_{t T} \neq 0 \Rightarrow \sigma_{T}=-\mathcal{F}\left|\sigma_{N}\right| \frac{u_{t T}}{\left|u_{t T}\right|}
\end{array}
$$

Here we denote [2]: $u_{N}=u_{i} n_{i}, \sigma_{N}=\sigma_{i j} n_{i} n_{j},\left(u_{T}\right)_{i}=u_{i}-u_{N} n_{i},\left(\sigma_{T}\right)_{i}=$ $\sigma_{i j} n_{j}-\sigma_{N} n_{i} i, j=1,2, n=\left(n_{1}, n_{2}\right)$ is the unit outward versor to the boundary $\Gamma . \mathcal{F}=\mathcal{F}\left(x, u_{t}\right)=\mathcal{F}\left(u_{t}\right)$ denotes a friction coefficient. The initial conditions are:

$$
u_{i}(0, x)=u_{0} \quad u_{t i}(0, x)=u_{1}, \quad i=1,2, \quad x \in \Omega
$$

where $u_{0}$ and $u_{1}$ are given functions. We shall consider problem (2) $-(7)$ in the variational form. Assume,

$$
\begin{array}{r}
f \in H^{1 / 4}\left(I ;\left(H^{1}\left(\Omega ; R^{2}\right)\right)^{*}\right) \cap L^{2}\left(Q ; R^{2}\right), \\
p \in L^{2}\left(I ;\left(H^{1 / 2}\left(\Gamma_{1} ; R^{2}\right)\right)^{*}\right), \\
u_{0} \in H^{3 / 2}\left(\Omega ; R^{2}\right) \quad u_{1} \in H^{3 / 2}\left(\Omega ; R^{2}\right), \quad u_{1 \mid \Gamma_{2}}=0,
\end{array}
$$

are given. The space $L^{2}\left(Q ; R^{2}\right)$ and the Sobolev spaces $H^{1 / 2}\left(\Gamma_{1} ; R^{2}\right)$ as well as $H^{1 / 4}\left(I ;\left(H^{1}\left(\Omega ; R^{2}\right)\right)^{*}\right)$ are defined in [1]. Note, that from (8) as well as from Sobolev Imbedding Theorem [1] it follows that $u_{0}$ and $u_{1}$ in (7) are continuous on the boundary of cylinder $Q$. The friction coefficient $\mathcal{F}: \Gamma_{2} \times R_{+} \rightarrow R_{+}$, $\mathcal{F} \in L^{\infty}\left(\Gamma_{2} ; R^{2}\right)$ has the following properties:

$$
\begin{array}{r}
\left|\mathcal{F}\left(x, u_{1}\right)-\mathcal{F}\left(x, u_{2}\right)\right| \leq L\left|u_{1}-u_{2}\right| \forall u_{1}, u_{2} \in[0,+\infty) \text { a.e. } x \in \Gamma_{2}, \\
0 \leq \mathcal{F}(x, u) \leq \mathcal{F}_{0} \text { a.e. } x \in \Gamma_{2}, \quad \forall u \in R_{+},
\end{array}
$$


and the function $x \rightarrow \mathcal{F}(x, u)$ is measurable for $u \in R_{+}$. We shall write $\mathcal{F}=\mathcal{F}\left(u_{t}\right)=\mathcal{F}\left(x, u_{t}\right)$. Let us introduce:

$$
F=\left\{z \in L^{2}\left(I ; H^{1}\left(\Omega ; R^{2}\right)\right): z_{i}=0 \text { on }(0, \mathcal{T}) \times \Gamma_{0}, i=1,2\right\},
$$

and the kinematically admissible set

$$
K=\left\{z \in F: z_{t}=0 \text { on }(0, \mathcal{T}) \times \Gamma_{0}, z_{t N} \leq 0 \quad \text { on }(0, \mathcal{T}) \times \Gamma_{2}\right\} .
$$

The problem (1) - (7) is equivalent to the following variational problem [3]: find a function $u \in L^{\infty}\left(I ; H^{1}\left(\Omega ; R^{2}\right)\right) \cap H^{1 / 2}\left(I ; L^{2}\left(\Omega ; R^{2}\right)\right) \cap K$ such that $u_{t} \in$ $L^{\infty}\left(I ; L^{2}\left(\Omega ; R^{2}\right)\right) \cap H^{1 / 2}\left(I ; L^{2}\left(\Omega ; R^{2}\right)\right) \cap K$ and $u_{t t} \in L^{\infty}\left(I ; H^{-1}\left(\Omega ; R^{2}\right)\right)$ $\cap\left(H^{1 / 2}\left(I ; L^{2}\left(\Omega ; R^{2}\right)\right)\right)^{*}$ satisfying the following inequality,

$$
\begin{array}{r}
\int_{Q} u_{t t i}\left(v_{i}-u_{t i}\right) d x d \tau+\int_{Q} \sigma_{i j}(u) e_{i j}\left(v_{i}-u_{t i}\right) d x d \tau+ \\
\int_{\gamma_{2}} \mathcal{F}\left|\sigma_{N}(u)\right|\left(\left|v_{T}\right|-\left|u_{t T}\right|\right) d x d \tau \geq \int_{Q} f_{i}\left(v_{i}-u_{t i}\right) d x d \tau+ \\
\int_{\gamma_{1}} p_{i}\left(v_{i}-u_{t i}\right) d x d \tau \quad \forall v \in H^{1 / 2}\left(I ; H^{1}\left(\Omega ; R^{2}\right)\right) \cap K .
\end{array}
$$

The existence of solutions to system (1) - (7) was shown in [3, Theorem 5.1.8, p. 286]:

THEOREM 1 Assume : (i) condition (8) holds, (ii) $\Gamma_{2}$ is of class $C^{\beta}, \beta>2$, (iii) tensors $c_{i j k l}^{\eta}(x), \eta=0,1$, are Hölder continuous with respect to $x$ with $\beta^{\prime}>1 / 2$, (iv) the friction coefficient $\mathcal{F}$ is bounded. Than there exists a weak solution to the problem (1) - (7).

Proof. The proof is based on penalization of the inequality (11), friction regularization and employment of localization and shifting technique due to Lions and Magenes. For details of the proof see [3].

REMARK 2 Assuming that $\mathcal{F}$ satisfies the conditions (9), (10) and using the same arguments as in proof of Theorem 2.1 in [5] one can prove that the solution to (11) is unique.

For the sake of brevity we shall consider the contact problem with the prescribed friction, i.e., we shall assume $g_{N} \in L^{2}\left(I ;\left(H^{1 / 2}\left(\Gamma_{2}\right)\right)^{\star}\right)$ is a given normal traction and

$$
\left|\sigma_{N}\right|=g_{N}, \quad \mathcal{F}\left(u_{t}\right)\left|g_{N}\right|=\sigma_{T} \leq 1 .
$$

The condition (6) is replaced by the following one,

$$
u_{t T} \sigma_{T}+\mathcal{F} g_{N}\left|u_{t T}\right|=0, \quad\left|\sigma_{T}\right| \leq 1 \quad \text { on } I \times \Gamma_{2} .
$$


Let us introduce the set,

$$
\Lambda=\left\{\lambda \in L^{\infty}\left(I ; L^{2}\left(\Gamma_{2}\right)\right):|\lambda| \leq 1 \quad \text { on } I \times \Gamma_{2}\right\} .
$$

Taking into account (15) the system (11) takes the form: find $u \in K$ and $\lambda \in \Lambda$ such that,

$$
\begin{array}{r}
\int_{Q} u_{t t i}\left(v_{i}-u_{t i}\right) d x d \tau+\int_{Q} \sigma_{i j}(u) e_{i j}\left(v_{i}-u_{t i}\right) d x d \tau- \\
\int_{\gamma_{2}} \mathcal{F} g_{N} \lambda_{T}\left(v_{T}-u_{t T}\right) d x d \tau \geq \int_{Q} f_{i}\left(v_{i}-u_{t i}\right) d x d \tau+ \\
\int_{\gamma_{1}} p_{i}\left(v_{i}-u_{t i}\right) d x d \tau \quad \forall v \in H^{1 / 2}\left(I ; H^{1}\left(\Omega ; R^{2}\right)\right) \cap K, \\
\int_{\gamma_{2}} \sigma_{T} u_{t T} d s d \tau \leq \int_{\gamma_{2}} \mathcal{F} g_{N} \lambda_{T} u_{t T} d s d \tau \quad \forall \lambda_{T} \in \Lambda .
\end{array}
$$

\section{Formulation of the shape optimization problem}

Consider a family $\left\{\Omega_{s}\right\}$ of the domains $\Omega_{s}$ depending on a parameter $s$. The domain $\Omega_{s}$ we shall consider as an image of a reference domain $\Omega$ under a smooth mapping $T_{s}$. To describe the transformation $T_{s}$ we shall use the speed method [11]. Let us denote by $V(.,):.(0, \vartheta) \times R^{2} \rightarrow R^{2}$ enough regular vector field depending on the parameter $s \in[0, \vartheta), \vartheta>0$ :

$$
V(s, .) \in C^{2}\left(R^{2}, R^{2}\right) \forall s \in[0, \vartheta), \quad V(., x) \in C\left([0, \vartheta), R^{2}\right) \forall x \in R^{2} .
$$

Let $T_{s}(V): R^{2} \ni X \rightarrow x(t, X) \in R^{2}$ denotes the family of mappings depending on a velocity field $V$. The vector function $x(., X)=x($.$) satisfies$ the systems of ordinary differential equations:

$$
\frac{d}{d \tau} x(\tau, X)=V(\tau, x(\tau, X)), \tau \in[0, \vartheta), \quad x(0, X)=X \in R .
$$

The family of domains $\left\{\Omega_{s}\right\}$ depending on parameter $s \in[0, \vartheta), \vartheta>0$, is defined as follows: $\Omega_{0}=\Omega$, and $\Omega_{s}=T_{s}(\Omega)(V)=\left\{x \in R^{2}: \exists X \in R^{2}\right.$ such that, $x=x(s, X)$, where the function $x(., X)$ satisfies equation (18) for $0 \leq \tau \leq s\}$.

Consider problem (17) - (18) in the domain $\Omega_{s}$. Let $F_{s}, K_{s}, \Lambda_{s}$ be defined, respectively, by $\left(9,(10)\right.$, (16) with $\Omega_{s}$ rather than $\Omega$. We shall write $u_{s}=u\left(\Omega_{s}\right), \sigma_{s}=\sigma\left(\Omega_{s}\right)$. The problem (17) - (18) in the domain $\Omega_{s}$ takes the form: find $u_{s} \in L^{\infty}\left(I ; H^{1}\left(\Omega_{s} ; R^{2}\right)\right) \cap H^{1 / 2}\left(I ; L^{2}\left(\Omega_{s} ; R^{2}\right)\right) \cap K$ such that $u_{t s} \in L^{\infty}\left(I ; L^{2}\left(\Omega_{s} ; R^{2}\right)\right) \cap H^{1 / 2}\left(I ; L^{2}\left(\Omega_{s} ; R^{2}\right)\right) \cap K$ and $u_{t t s} \in$ 


$$
\begin{gathered}
L^{\infty}\left(I ; H^{-1}\left(\Omega_{s} ; R^{2}\right)\right) \cap\left(H^{1 / 2}\left(I ; L^{2}\left(\Omega_{s} ; R^{2}\right)\right)\right)^{*} \text { and } \lambda_{s} \in \Lambda_{s} \text { such that, } \\
\int_{Q_{s}} u_{t t s i} v_{i} d x d \tau+\int_{Q_{s}} \sigma_{i j}\left(u_{s}\right) e_{i j}\left(v_{i}-u_{t s i}\right) d x d \tau- \\
\int_{\gamma_{s 2}} \mathcal{F}_{s} g_{N} \lambda_{s}\left(v_{T}-u_{t s T}\right) d x d \tau \geq \int_{Q_{s}} f_{i}\left(v_{i}-u_{t s i}\right) d x d \tau+ \\
\int_{\gamma_{s 1}} p_{i}\left(v_{i}-u_{t s i}\right) d x d \tau \quad \forall v \in H^{1 / 2}\left(I ; H^{1}\left(\Omega_{s} ; R^{2}\right)\right) \cap K \\
\int_{\gamma_{s 2}} \sigma_{s T} u_{t s T} d s d \tau \leq \int_{\gamma_{s 2}} \mathcal{F}_{s} g_{N} \lambda_{s} u_{t s T} d s d \tau \quad \forall \lambda_{s} \in \Lambda_{s} .
\end{gathered}
$$

Let us formulate the optimization problem. By $\hat{\Omega} \subset R^{2}$ we denote a hold all domain such that $\Omega_{s} \subset \hat{\Omega}$ for all $s \in[0, \vartheta), \vartheta>0$ and by $P_{\hat{\Omega}}(\Omega)$ a finite perimeter [11] of a domain $\Omega$ in $\hat{\Omega}$. Let $M$ be an auxiliary set $\mathrm{M}$ determined as $M=\left\{\phi \in L^{\infty}\left(I ; H_{0}^{2}\left(\hat{\Omega} ; R^{2}\right): \phi \leq 0\right.\right.$ on $\left.I \times \hat{\Omega},\|\phi\|_{L^{\infty}\left(I ; H_{0}^{2}\left(\hat{\Omega} ; R^{2}\right)\right.} \leq 1\right\}$. Introduce, for a given $\phi \in M$, the following cost functional:

$$
J_{\phi}\left(u_{s}\right)=J_{\phi}^{1}\left(u_{s}\right)+P_{\hat{\Omega}}(\Omega) \text {, where } J_{\phi}^{1}\left(u_{s}\right)=\int_{\gamma_{s 2}} \sigma_{s N} \phi_{t N s} d z d \tau \text {, }
$$

and $\phi_{t N s}$ and $\sigma_{s N}$ are normal components of $\phi_{t s}$ and $\sigma_{s}$, respectively, depending on a parameter $s$. The cost functional (23) depends on the solution $\left(u_{s}, \lambda_{s}\right) \in$ $K_{s} \times \Lambda_{s}$ of the system (21) - (22).

We shall consider such family of domains $\left\{\Omega_{s}\right\}$, that every $\Omega_{s}, s \in[0, \vartheta), \vartheta>$ 0 , has a constant volume $c>0$, i.e., every $\Omega_{s}$ belongs to the constraint set $\mathrm{U}$ given by:

$$
U=\left\{\Omega_{s}: \int_{\Omega_{s}} d x=c\right\}
$$

The set $U$ is assumed to be nonempty. The shape optimization problem has the form:

For a given $\phi \in M$, find a domain $\Omega_{s}$ occupied by the body, minimizing the cost functional (23) subject to $\Omega_{s} \in U$.

The goal of the shape optimization problem (22) is to find such boundary $\Gamma_{2}$ of the domain $\Omega$ occupied by the body that the normal contact stress is minimized. It is known [4] that the normal contact stress attains peak in the contact area. This stress can be significantly reduced and uniformly distributed when the bodies in cantact have an optimal shape. The finite perimeter term in (23) is added to ensure the existence of solutions to the shape optimization problem (22). This term implies the compactness of the set (24) in $L^{2}$ topology of its characteristic functions. For detailed discussion concerning the conditions ensuring the existence of optimal solutions to shape optimization problems see $[4,11]$. 


\section{Shape derivatives of contact problem solution}

In order to calculate Euler derivative (42) of the cost functional (23) we have to determine material $(\dot{u}, \dot{\lambda}) \in F \times \Lambda$ and shape derivatives $\left(u^{\prime}, \lambda^{\prime}\right) \in F \times \Lambda$ of a solution $\left(u_{s}, \lambda_{s}\right) \in K_{s} \times \Lambda_{s}$ of the system (21)-(22). Recall the notion of the material derivative [11]:

DEFINITION 3 The material derivative $\dot{u} \in F$ of the function $u_{s} \in F_{s}$ at a point $X \in \Omega$ is determined by :

$$
\lim _{s \rightarrow 0}\left\|\left[\left(u_{s} \circ T_{s}\right)-u_{0}\right] / s-\dot{u}\right\|_{F}=0,
$$

where $u_{0} \in F, u_{s} \circ T_{s} \in F$ is an image of function $u_{s} \in F_{s}$ in the space $F$ under the mapping $T_{s}$.

Recall [11], that if the shape derivative $u^{\prime} \in F$ of the function $u_{s} \in F_{s}$ exists, then it holds:

$$
u^{\prime} \stackrel{\text { def }}{=} \dot{u}-\nabla u V(0)
$$

where $\dot{u} \in F$ is material derivative of the function $u_{s} \in F_{s}$. Taking into account Definition 3 we can calculate the material derivative of a solution to the system (21), (22):

LEMMA 4 The material derivatives $(\dot{u}, \dot{\lambda}) \in K_{1} \times \Lambda$ of a solution $\left(u_{s}, \lambda_{s}\right) \in$ $K_{s} \times \Lambda_{s}$ to the system (21) - (22) are determined as a unique solution to the following system:

$$
\begin{aligned}
& \int_{Q}\left\{\left(\dot{u}_{t t} \eta+u_{t t} \dot{\eta}+u_{t t} \eta d i v V(0)+(D V(0) u)_{t t} \eta+u_{t t}(D V(0) \eta)\right.\right. \\
& \left.-\dot{f} \eta-f \dot{\eta}+\left(\sigma_{i j}(u) e_{k l}(\eta)-f \eta\right) d i v V(0)\right\} d x d \tau- \\
& \int_{\gamma_{1}}(\dot{p} \eta+p \dot{\eta}+p \eta D) d x d \tau-\int_{\gamma_{2}}\left\{\left(\frac{\partial \mathcal{F}}{\partial u_{t}} \dot{u}_{t} g_{N} \lambda \eta_{t T}+\mathcal{F} g_{N} \dot{\lambda} \eta_{t T}\right)+\right. \\
& \left.\left(\mathcal{F} g_{N} \lambda \dot{\eta}_{t T}+\mathcal{F} g_{N} \lambda \eta_{t T} D\right)\right\} d x d \tau \geq 0 \quad \forall \eta \in K_{1} \\
& \int_{\gamma_{2}}\left(\left(\frac{\partial \mathcal{F}}{\partial u_{t}} \dot{u}_{t} g_{N} \lambda+\mathcal{F} g_{N} \dot{\lambda}-\mu\right) u_{t T}+\left(\mathcal{F} g_{N} \lambda-\dot{\mu}\right) u_{t T}+\right. \\
& \left.\left(\mathcal{F} g_{N} \lambda-\mu\right) \dot{u}_{t T}+\mathcal{F} \lambda u_{t T} D\right\} g_{N} d x d \tau \quad \forall \mu \in L_{1}
\end{aligned}
$$

where $V(0)=V(0, X), D V(0)$ denotes the Jacobian of the matrix $V(0)$ and $D$ is the expression

$$
D=\operatorname{div} V(0)-(D V(0) n, n) .
$$

Moreover the sets $K_{1}$ and $L_{1}$ are determined by

$$
K_{1}=\left\{\xi \in F: \xi=u-D V u \text { on } \gamma_{0}, \begin{array}{l}
\xi n \geq n D V(0) u \text { on } A_{1}, \\
\left.\xi n=n D V(0) u \text { on } A_{2}\right\},
\end{array}\right.
$$




$$
L_{1}=\left\{\xi \in \Lambda: \xi \geq 0 \text { on } B_{2}, \xi \leq 0 \text { on } B_{1}, \xi=0 \text { on } B_{0}\right\},
$$

where the sets $A_{i}, B_{i}, i=0,1,2$ are given by: $A_{0}=\left\{x \in \gamma_{2}: u_{t N}=0\right\}$, $A_{1}=\left\{x \in A_{0}: \sigma_{N}=0\right\}, A_{2}=\left\{x \in A_{0}: \sigma_{N}<0\right\}, B_{0}=\{x \in$ $\left.\gamma_{2}: \lambda_{T}=1, \quad u_{t T} \neq 0\right\}, B_{1}=\left\{x \in \gamma_{2}: \lambda_{T}=-1, \quad u_{t T}=0\right\}$, $B_{2}=\left\{x \in \gamma_{2}: \lambda_{T}=1,: u_{t T}=0\right\}$.

Proof: is based on approach proposed in [11]. First we transport the system (21) - (22) to the fixed domain $\Omega$. Let $u^{s}=u_{s} \circ T_{s} \in F, u=u_{0} \in F$, $\lambda^{s}=\lambda_{s} \circ T_{s} \in \Lambda, \lambda=\lambda_{0} \in \Lambda$. Since in general $u^{s} \notin K(\Omega)$ we introduce a new variable $z^{s}=D T_{s}^{-1} u^{s} \in K$. Moreover $\dot{z}=\dot{u}-D V(0) u$ [11]. Using this new variable $z^{s}$ as well as the formulae for transformation of the function and its gradient into reference domain $\Omega$ [11] we write the system (21) - (22) in the reference domain $\Omega$. Using the estimates on time derivative of function $u$ [11] the Lipschitz continuity of $u$ and $\lambda$ satisfying (21) - (22) with respect to $s$ can be proved. Applying to this system the result concerning the differentiability of solutions to variational inequality [11] we obtain that the material derivative $(\dot{u}, \dot{\lambda}) \in K_{1} \times \Lambda$ satisfies the system (28) - (29). Moreover from the ellipticity condition of the elasticity coefficients by a standard argument [11] it follows that $(\dot{u}, \dot{\lambda}) \in K_{1} \times \Lambda$ is a unique solution to the system (28) - (29).

Assume

$$
\nabla u V(0) \in F, \quad \nabla \lambda_{T} V(0) \in \Lambda,
$$

where the spaces $F$ and $\Lambda$ are determined by (9) and (16) respectively. Integrating by parts system (28), (29) and taking into account (27), (33) we obtain the similar system to (28) - (29) determining the shape derivative $\left(u^{\prime}, \lambda_{T}^{\prime}\right) \in F \times L$ of the solution $\left(u_{s}, \lambda_{s T}\right) \in K_{s} \times L_{s}$ of the system (21) - (22):

$$
\begin{gathered}
\int_{Q}\left[u_{t t}^{\prime} \eta+u_{t t} \eta^{\prime}+\left(D V(0)+{ }^{*} D V(0)\right) u_{t t} \eta\right] d x d \tau+ \\
\int_{\gamma} u_{t t} \eta V(0) n d x d \tau+\int_{Q} \sigma_{i j}\left(u^{\prime}\right) e_{k l} \eta d x d \tau- \\
\int_{\gamma_{2}}\left\{\frac{\partial \mathcal{F}}{\partial u_{t}} u_{t}^{\prime} g_{n} \lambda \eta_{t T}+\mathcal{F} g_{N} \lambda^{\prime} \eta_{t T}+\mathcal{F} g_{N} \lambda \eta_{t T}^{\prime}\right\} d x d \tau+ \\
I_{1}\left(u_{t}, \eta\right)+I_{2}(\lambda, u, \eta) \geq 0 \quad \forall \eta \in N_{1}, \\
\int_{\gamma_{2}}\left[\frac{\partial \mathcal{F}}{\partial u_{t}} u_{t}^{\prime} g_{n} \lambda u_{t T} u_{t T}^{\prime}\left(\mu-\mathcal{F} g_{N} \lambda\right)-u_{t T} \mathcal{F} g_{N} \lambda^{\prime}\right] d x d \tau+ \\
I_{3}(u, \mu-\lambda) \geq 0 \quad \forall \mu \in L_{1}, \\
N_{1}=\left\{\eta \in F: \eta=\lambda-D u V(0), \quad \lambda \in K_{1}\right\}, \\
I_{1}(\varphi, \phi)=\int_{\gamma}\left\{\sigma_{i j}(\varphi) e_{k l} \phi-f \phi-\right.
\end{gathered}
$$




$$
\begin{gathered}
((\nabla p n) \phi+(p \nabla \phi) n+p \phi \kappa) V(0) n\} d x d \tau \\
I_{2}(\mu, \varphi, \phi)=\int_{\gamma_{2}}\left\{\frac{\partial \mathcal{F}}{\partial \varphi_{t}} \nabla \varphi_{t} g_{n} \mu \varphi_{t T}+\left(\nabla \mu \mathcal{F} g_{N}\right) n \nabla \phi+\right. \\
\left.\mathcal{F} g_{N} \mu(\nabla(\nabla \varphi n)) \varphi+\mathcal{F} g_{N} \mu \nabla \varphi_{t T} H+\mathcal{F} g_{N} \mu \nabla \varphi n\right\} V(0) n d x d \tau \\
I_{3}(\varphi, \mu-\lambda)=\int_{\gamma_{2}}\left[\frac{\partial \mathcal{F}}{\partial \varphi_{t}} \nabla \varphi_{t} g_{n} \mu+(\varphi n)\left(\mu-\mathcal{F} g_{N} \lambda\right)+\varphi(\nabla \mu n)-\right. \\
\left.\varphi\left(\nabla \mathcal{F} g_{N} \lambda n\right)+\varphi\left(\mu-\mathcal{F} g_{N} \lambda\right) \kappa\right] V(0) n d x d \tau
\end{gathered}
$$

where $\kappa$ denotes a mean curvature of the boundary $\Gamma$ [11].

\section{Necessary optimality condition}

Our goal is to calculate the directional derivative of the cost functional (23) with respect to the parameter $s$. We will use this derivative to formulate a necessary optimality condition for the optimization problem (22). First, recall from [11] the notion of Euler derivative of the cost functional depending on a domain $\Omega$ :

DEFINITION 5 Euler derivative $d J(\Omega ; V)$ of the cost functional $J$ at a point $\Omega$ in the direction of the vector field $V$ is given by:

$$
d J(\Omega ; V)=\limsup _{s \rightarrow 0}\left[J\left(\Omega_{s}\right)-J(\Omega)\right] / s .
$$

By direct application of this Definition we get the form of the directional derivative $d J_{\phi}(u ; V)$ of the cost functional (23):

LEMMA 6 The directional derivative $J_{\phi}(u ; V)$ of the cost functional (23), for $\phi \in M$ given, at a point $u \in K$ in the direction of vector field $V$ is determined by:

$$
\begin{array}{r}
d J_{\phi}(u ; V)=\int_{Q}\left[u_{t t}^{\prime} \eta+u_{t t} \eta^{\prime}+\left(D V(0)+{ }^{*} D V(0)\right) u_{t t} \eta\right] d x d \tau+ \\
\int_{\gamma} u_{t t} \eta V(0) n d x d \tau+\int_{Q}\left(\sigma_{i j}^{\prime} e_{k l} l \phi\right) d x d \tau+ \\
\int_{\Gamma}\left(\sigma_{i j} e_{k l}(\phi)-f \phi\right) V(0) n d s-\int_{\Gamma_{1}}[\nabla p \phi V(0)+ \\
p \nabla \phi V(0)+p \phi D)] d s-\int_{\Gamma_{2}} \lambda^{\prime} \phi_{T} d s+I_{1}(u, \phi)-I_{2}(\lambda, u, \phi),
\end{array}
$$

where $\sigma^{\prime}$ is a shape derivative of the function $\sigma_{s}$ with respect to $s . \nabla p$ is a gradient of function $p$ with respect to $x$. Moreover $V(0)=V(0, X), \phi_{T}$ and 
$\sigma_{T}$ are tangent components of $\phi$ and $\sigma$, respectively, as well as $D$ is given by (30). DV (0) denotes the Jacobian of the matrix $V(0)$.

In order to eliminate the shape derivative $\left(u^{\prime}, \lambda^{\prime}\right)$ from (42) we introduce an adjoint state $(r, q) \in K_{2} \times L_{2}$ defined as follows:

$$
\begin{gathered}
\int_{Q} r_{t t} \zeta d x d \tau+\int_{Q} \sigma_{i j}(\zeta) e_{k l}(\phi+r) d x d \tau+ \\
\int_{\gamma_{2}} \frac{\partial \mathcal{F}}{\partial u_{t}} g_{N} r_{t T}(q-\lambda) \zeta d x d \tau=0, \quad \forall \zeta \in K_{2}
\end{gathered}
$$

with

$$
\begin{gathered}
r(\mathcal{T}, x)=0, \quad r_{t}(\mathcal{T}, x)=0 \\
\int_{\gamma_{2}} \mathcal{F}_{g_{N}}\left(r_{t T}+\phi_{t T}-u_{t T}\right) \delta d x d \tau=0, \quad \forall \delta \in L_{2}
\end{gathered}
$$

where $K_{2}=\left\{\zeta \in K_{1}: \zeta n=0\right.$ on $\left.A_{0}\right\}$ and $L_{2}=\{\delta \in \Lambda: \delta=$ 0 on $\left.A_{0} \cap B_{0}\right\}$. Since $\phi \in M$ is a given element, then by the same arguments as used to show the existence of the solution $(u, \lambda) \in K \times L$ to the system (21)-(22) we can show the existence of the solution $(r, q) \in K_{2} \times L_{2}$ to the system (43) - (44). From (42), (34), (35), (43), (44) we obtain:

$$
d J_{\phi}(u ; V)=I_{1}(u, \phi+r)+I_{2}(\lambda, u, \phi+r)+I_{3}(u, q-\lambda) .
$$

The necessary optimality condition has a standard form $[2,9,11]$ :

THEOREM 7 Let $\Omega^{\star} \subset U$ be an optimal solution to the problem (22). Then there exists Lagrange multiplier $\mu \in R$ such that for all vector fields $V$ determined by (17), (18) the following condition holds:

$$
d J_{\phi}\left(u^{\star} ; V\right)+\mu \int_{\Gamma^{\star}} V(0) n d s+d P_{\hat{\Omega}}\left(\Omega^{\star} ; V\right) \geq 0,
$$

where $d J_{\phi}\left(u^{\star} ; V\right)$ is given by (45), $\Gamma^{\star}$ denotes the boundary of the optimal domain $\Omega^{\star}, u^{\star}$ is a solution to the state system $(17)-(18)$ in $\Omega^{\star}$, and $d P_{\hat{\Omega}}\left(\Omega^{\star} ; V\right)$ denotes Euler derivative of $P_{\hat{\Omega}}(\Omega)$ at $\Omega^{\star}$ in a direction $V$.

\section{Conclusions}

The directional derivative of the cost functional with respect to the perturbation of the domain occupied by a viscoelastic body in unilateral contact with a rigid foundation was calculated and the necessary optimality condition for the shape optimization problem for the dynamical contact problem was formulated. This shape optimization problem has been numerically solved. Preliminary numerical results can be found in [10]. 


\section{References}

[1] R.A. Adams. Sobolev Spaces, Academic Press, New York, 1975.

[2] G. Duvaut and J.L. Lions. Les inequations en mecanique et en physique, Dunod, Paris, 1972.

[3] C. Eck, J. Jarusek and M. Krbec. Unilateral Contact Problems. Variational Methods and Existence Theorems, CRC Press, Boca Raton, Florida, USA, 2005.

[4] J. Haslinger, P. Neittaanmaki. Finite Element Approximation for Optimal Shape Design. Theory and Application., John Wiley\& Sons, 1988.

[5] I. Ionescu. Viscosity Solutions for Dynamic Problems with Slip - Rate Dependent Friction, Quarterly of Mathematics, LX:461 - 476, 2002.

[6] J. Jarušek, M. Krbec, M. Rao, J. Sokołowski. Conical Differentiability for Evolution Variational Inequalities, University of Nancy I, 2002.

[7] K.L. Kuttler, M. Schillor. Regularity of Solutions to a Dynamic Frictionless Contact Problem with Normal Compliance, Nonlinear Analysis, 59:1063 - 1075, 2004.

[8] K.L. Kuttler, M. Schillor, Dynamic Contact with Signorini's Condition and Slip Rate Dependent Friction, Electronic Journal of Differential Equations, 1 - 21, 2004.

[9] A. Myśliński. Shape Optimization for Dynamic Contact Problems, Discussiones Mathematicae, Differential Inclusions, Control and Optimization, 20:79 - 91, 2000.

[10] A. Myśliński. Augmented Lagrangian Techniques for Shape Optimal Design of Dynamic Contact Problems, CD - ROM Proceedings of the Fourth World Congress on Structural and Multidisciplinary Optimization, G. Chen, Y. Gu, S. Liu, Y. Wang eds., Liaoning Electronic Press, Shenyang, China, 2001.

[11] J. Sokołowski and J.P. Zolesio. Introduction to Shape Optimization. Shape Sensitivity Analysis. Springer, Berlin, 1992. 